\title{
Studies in auditory timing: 2. Rhythm patterns
}

\author{
CAROLINE B. MONAHAN and IRA J. HIRSH \\ Central Institute for the Deaf, St. Louis, Missouri
}

\begin{abstract}
Listeners discriminated between 6-tone rhythmic patterns that differed only in the delay of the temporal position of one of the tones. On each trial, feedback was given and the subject's performance determined the amount of delay on the next trial. The 6 tones of the patterns marked off 5 intervals. In the first experiment, patterns comprised 3 "short" and 2 "long" intervals: 12121, 21121, and so forth, where the long (2) was twice the length of a short (1). In the second experiment, patterns were the complements of the patterns in the first experiment and comprised 2 shorts and 3 longs: 21212,12212 , and so forth. Each pattern was tested 45 times (5 positions of the delayed tone $\times 3$ tempos $\times 3$ replications). Consistent with previous work on simple interval discrimination, absolute discrimination ( $\Delta t$ in milliseconds) was poorer the longer the intervals (i.e., the slower the tempo). Measures of relative discrimination ( $\Delta t / t$, where $t$ was the short interval, the long interval, or the average of 2 intervals surrounding the delayed tone) were better the slower the tempo. Beyond these global results, large interactions of pattern with position of the delayed tone and tempo suggest that different models of performance are needed to explain behavior at the different tempos. A Weber's law model fit the slow-tempo data better than did a model based on positions of "natural accent" (Povel \& Essens, 1985).
\end{abstract}

In the preceding study in this series on timing discrimination, Hirsh, Monahan, Grant, and Singh (1990) found that temporal interval discrimination for tonal onsets in a sequence of tones was similar to older results for the discrimination of single temporal intervals (Abel, 1972; Creelman, 1962; Getty, 1975; Small \& Campbell, 1962). The relative difference limen (DL; $\Delta t / t)$ is fairly constant for intervals from 100 to $1,500 \mathrm{msec}$, becomes larger for shorter intervals, and may even get better for longer intervals up to $8 \mathrm{sec}$ (Killeen \& Weiss, 1987).

A listener's accuracy in discriminating a small delay in the temporal position of a tone in a sequence is thus clearly related to the average interval separating the tones or (reciprocally) to the tempo. Furthermore, when one of the tones in a sequence is of a different pitch, or when one of the intervals is made longer than the others, discrimination of timing is somewhat more difficult in the vicinity of the changed tone or interval (Hirsh et al., 1990). In the case of the longer interval, the change in performance might reflect the same relative discrimination near a longer standard. No such explanation applies to a pitch change, however.

In rhythmic structures, certain elements seem to be accented. Accents may be generated by pitch changes or

This research was supported by Air Force Grant AFOSR-87-0382 to the Central Institute for the Deaf, and by Washington University, where Ira Hirsh is Distinguished University Professor. Thanks are extended to Julius Goldstein, Stephen Handel, and an anonymous reviewer for comments on an earlier version of the manuscript. We especially thank Joanne Kocunik and Jay Babcock for assistance in the experiments. Correspondence may be addressed to Caroline B. Monahan or Ira J. Hirsh, Central Institute for the Deaf, 818 South Euclid, St. Louis, MO 63110. by longer temporal intervals, among other possibilities (Monahan \& Carterette, 1985; Monahan, Kendall, \& Carterette, 1987). Perhaps pitch changes and interval accents play a similar role. Are accented tones, from either source, easier or harder to discriminate temporally?

\section{Accenting and Pitch}

Hirsh et al. (1990) observed that large pitch changes had a more disruptive effect on timing discrimination than did small ones. Might this have something to do with the fact that pitch intervals of more than 5 semitones (a musical fourth, or about $1 / 3$ octave) are exceedingly rare in the world's music, constituting less than $10 \%$ of intervals in the literature surveyed (Dowling, 1968)? Dowling and Harwood (1986) have called this finding a musical universal, and suggest that it reflects a basic property of the auditory system. Tones that are more than 4 or 5 semitones apart will tend to fall in different critical bands and will thus be less likely to mask one another. Fitzgibbons, Pollatsek, and Thomas (1974) have also reported that the detection of temporal gaps between tones that were about $21 / 2$ octaves apart was very difficult. People notice gaps within frequency streams or groupings rather than between streams or groupings. Hirsh et al. expanded this result by showing that at a relatively fast musical tempo ( 5 tones $/ \mathrm{sec}$ or 300 beats $/ \mathrm{min}$ ), discrimination of the delay of a tone following a skip of 9 semitones was more difficult than discrimination of the delay of tones in a monotone series or after a pitch skip of only 2 semitones.

It also appears that tones that skip more than 4 or 5 semitones sound accented at musical tempos and sound especially accented if the tone marks a point of inflection in the pitch contour (Thomassen, 1982). Grant (1987) has 
shown that a rise of $F_{0}$ of 4 semitones is sufficient to cause a change in the perceived accent of a syllable in a sentence. Thomassen (1982), Monahan and Carterette (1985), and Monahan et al. (1987) have pointed out that tones that begin different auditory streams (Bregman \& Campbell, 1971; Dowling, 1968; van Noorden, 1975) at fast tempos (at 8 to 10 notes/sec) also tend to sound accented and begin new pitch groupings at somewhat slower musical tempos.

Thus, both relatively large pitch skips and pitch-contour inflections seem to reflect mechanisms of accent.

\section{Accenting and Temporal Intervals}

If pitch changes lead to greater temporal uncertainty by virtue of their establishing accents in a sequence, then we should be able to show similar effects through another source of accent. Among the various hypotheses about sources of accent, one depends solely on a change in timing. Tones in sequences appear to be accented (1) when the tone begins a long interval, and (2) to a lesser extent, when the tone ends a longer interval and begins a series of 3 or more intervals (Povel \& Okkerman, 1981). As an explanation for the first sort of accent, Povel and Okkerman rely on what might be termed a "release from backward masking" argument: the perceptual measurement of the relative strength of a tone takes time, and processing is interrupted when a new tone begins; therefore, the tone beginning a long interval is less subject to interference by the following tone(s), and sounds accented. As to the second sort of accent, the authors offer a "release from forward masking" argument: tones can interfere with each other if the time between the offset of one and the onset of the next is $250 \mathrm{msec}$ or less; therefore, tones that end a lengthened interval and begin a new series of 3 or more intervals are less subject to interference by the preceding tone(s), and may sound accented. The authors also suggest that there may be a "structural" basis, especially for the latter form of accent, that involves the hierarchical relations between beats and unaccented pulses (see section below) that has been exemplified previously only in repeated or cycled rhythmic patterns.

\section{Importance of the Regularity of Accenting}

One of the hypotheses that we consider below assumes that the listener carries a temporal referent of fixed length (a beat) against which to judge a single temporal change made in a pattern. Therefore, we feel it is appropriate to recount briefly some of the evidence that suggests that the presence of regular accenting (accenting that occurs at a fixed rate or interval) promotes both better perception and (re)production of auditory patterns. Martin (1972) first proposed that both speech and music could be described as having a hierarchical scheme with equally distributed accents, and showed in his experimental work (Shields, McHugh, \& Martin, 1974; Sturges \& Martin, 1974) that auditory patterns characterized by such a scheme were recognized both more accurately and more quickly than patterns that were not characterized by such a scheme.
Like Martin, other writers (Handel \& Lawson, 1983; Handel \& Oshinsky, 1981; Longuet-Higgins \& Lee, 1982; Monahan, 1984; Monahan \& Carterette, 1985; Monahan et al., 1987; Povel, 1984; Povel \& Essens, 1985; Steedman, 1977; Yeston, 1976) have assumed that time in Western music is psychologically arranged in a hierarchy as a rate or rates within a rate. That is, musical time may be characterized as having a slower, periodic accent rate, a metric, "clock," or beat, that is internalized by the listener. This clock is superimposed upon a temporal grain-a string of equal intervals (some of which are marked by note onsets and some of which are not).

Monahan and Carterette (1985, pp. 8-9) reviewed some experimental work favoring Martin's hypothesis. Other recent studies showing the importance of the distribution of accenting have been performed by Povel (1981), Povel and Essens (1985), Boltz and Jones (1986), Monahan et al. (1987), and Fourakis and Monahan (1988). The findings of Povel (1981) are most directly relevant to our experiments. Povel first asked his subjects to reproduce (by tapping) different series of two alternating interval durations. Like Fraisse (1963), Povel found that the only ratio of $t_{1}$ to $t_{2}$ (where $t_{1}$ is short and $t_{2}$ is long) that subjects accurately (re)produced was $1: 2$; other produced ratios tended to "drift" toward 1:2 or 1:1 ratios. Ratios of 1:3 were very accurately produced only in limited contextsfor example, where there were 4 repeating intervals in the ratios 1:1:1:3 (1113; e.g., 250-250-250-750 msec). Similarly, 1:4 ratios were accurately produced in the recycling pattern context 1:1:1:1:4 (11114; e.g., 250-250-250-250-1,000 msec). (In what follows, we will refer to time patterns in terms of the number of temporal grains or equal intervals between note onsets; thus, 1113 and 11114 are patterns of interonset intervals, or IOI patterns.) Povel (1981) suggested that what listeners hear in the pattern 1113 is a recurring temporal accent every $750 \mathrm{msec}$ : one temporal accent is for the tone beginning the long (3) interval and one is for the tone ending the long interval that begins the run of the 4 intervals as described in the preceding section. These accents demarcate 2 beat intervals: the first is filled with beat subdivisions that have a rate three times that of the beat rate, and the second beat interval is empty. On the other hand, Povel found that repeating patterns, such as $\mathbf{4 4 2}$ (e.g., $1,000-1,000-500 \mathrm{msec}$ ), were reproduced only with great difficulty and variability, presumably because the tone onsets that might be used to set up the perception of a regular beat in the listener are not equally distributed in time in such a pattern.

Western music favors the division of beats into two or three equal parts. Fraisse (1982) surveyed more than 50 pieces of Western classical music and found that most of the temporal values in any piece fell into two categories: long time values and short time values, where the long was either 2 or 3 times longer than the short, but the short occurred 2 or 3 times, respectively, more often than the long. In our series of studies, in order to make our patterns musically "sensible," we have restricted the 
ratios of temporal intervals in our standard patterns to $1: 1$ and $1: 2$.

\section{Alternative Hypotheses for Timing Discrimination}

If, in a sequence, the temporal position of tones marking the onset and offset of longer intervals is uncertain, one can also invoke Weber's law for explanation, as was done by Hirsh et al. (1990). There may be, however, still other features of the sequence, such as groupings, runs, and so forth, which do not necessarily point to accents yet may be related to temporal uncertainty. Our experiments are designed to afford an opportunity to test these various notions as they apply to timing discrimination.

\section{Purposes}

Accent versus the size of intervals. One main question, then, is whether, at musical tempos, the discriminability of delays of tones in rhythmic patterns can be better interpreted as a function of the position and regularity of "natural temporal accent" (Povel \& Essens, 1985) or, alternatively and more parsimoniously, as a function of the size of neighboring intervals in a pattern, as would be suggested by Weber's law.

Hirsh et al. (1990, Experiment 3) also raised this question, but were unable to suggest an answer, because, for the simple rhythmic sequences they employed, a model based on Weber's ratio and one based on the position of natural temporal accent would make almost exactly the same predictions with regard to temporal discriminability. The patterns we employ in the present experiments are sufficiently complicated to enable a choice between the predictions of an accent model or an interval-length (Weber) model.

Choice of procedure. A second question raised by Hirsh et al.'s (1990) Experiment 3 was one of procedure. How can we best measure absolute and relative temporal DLs at particular positions in a pattern? In our series of studies, the delay of a single tone relative to its position in a standard pattern concomitantly lengthens the interval preceding the tone and shortens the interval following the tone. Either interval may subserve the measure of absolute discriminability (in milliseconds). Note that our "delay-only" procedure differs from the "single gap discrimination" of Pollack (1967) and Bharucha and Pryor (1986), where an increase in the size of a single interval relative to the size of the corresponding interval in the standard sequence concomitantly changes the length of the sequence.

Measures of relative discrimination. A third related question raised by Hirsh et al.'s (1990) Experiment 3 concerns the measure of relative discrimination: What is the appropriate $t$ in the ratio $\Delta t / t$ when there are two different temporal intervals in a sequence? Should $t$ be the short or the long interval? Or, in order to control for differing note densities across patterns in different studies, should it be the average length of note intervals in a pattern? Or, since in our series of studies a single delayed tone lengthens the preceding interval and concomitantly shortens the following interval, should $t$ be based on the preceding interval, the following interval, or an average of the two? The first set of choices, using short, long, or average pattern interval as $t$, implies that the listener carries a fixed temporal referent against which to judge a delay at any point in the pattern. The second set of choices, using the preceding or following interval, or the average of the 2 intervals surrounding the delay, implies a process model of judgment that is dependent on the size of the intervals relative to the point of delay within the sequences. We will view our results with examples of both types of referent.

Unitization. A fourth concern of this paper is measuring temporal DLs in patterns at tempos where Weber's law is known to break down for single-interval discrimination. Tones seem to lose their individual quality at a presentation rate of 10/sec or faster (as in Pollack, 1967). If a pattern of tones is played at a high enough rate, the pattern itself is heard as a unit-a chunk of sound that comprises the whole cycle. Both Handel and Oshinsky (1981) and Royer and Robin (1986) reported that perceived unitization of repeating patterns occurred when the smallest IOI was about $100 \mathrm{msec}$ (or a rate of 10 tones $/ \mathrm{sec}$ ). Thus, we will measure temporal DLs both above and below this rate within different patterns.

Nature of the task and the amount of training. Two other concerns are more general in nature. The discriminability of temporal differences has typically been measured by the tapping responses of the subject. It has been argued that production or reproduction of rhythm patterns is based on perceptual and memory schema of temporal patterns (Povel, 1981). While this may be the case, production also involves the motor system. Thus, we have chosen here to have our subjects perform discrimination tasks that bypass the motor limitations that a tapping task may impose. Second, since we are interested in the limitations of the auditory system, we are primarily concerned with what listeners can do after a great deal of training and not what they may do on the spur of the moment. Furthermore, we try to present stimuli under optimum conditions for discriminating delay. In practice, this has meant that before every adaptive run of trials, the subjects are informed of the serial position of the tone to be delayed and they have indicated that they could hear a delay equivalent to that presented on the first trial of each run.

In two experiments, we explored the dependence of a listener's temporal precision on features of monotone sequences. In both experiments, we restricted the patterns to 6 tones separated by unequal intervals.

\section{EXPERIMENT 1}

\section{Patterns}

In Experiment 1, eight patterns had 2 long and 3 short intervals between 6 successive tones; a ninth pattern, 11211, had also been employed by Hirsh et al. (1990, Ex- 


\section{POSITION OF FIRST LONG INTERVAL}

DISTANCE
BETVEEN
"LONGS"
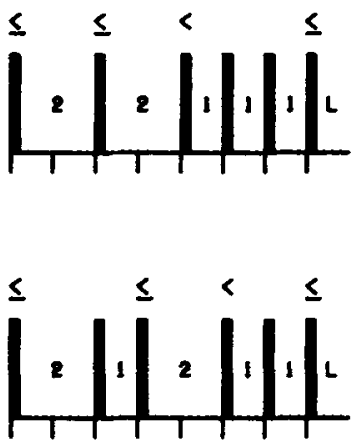

ONE

ZERO

- gHCRTs"

- SHVTt
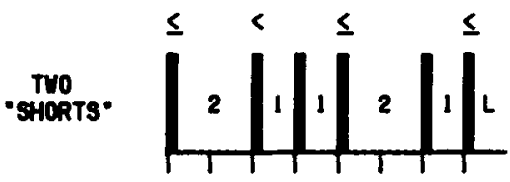

Tro
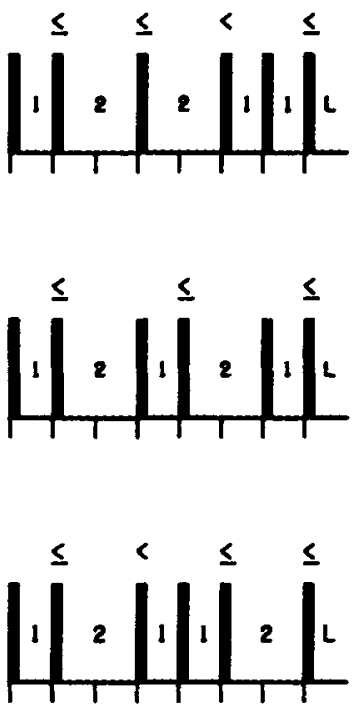

ThinEE
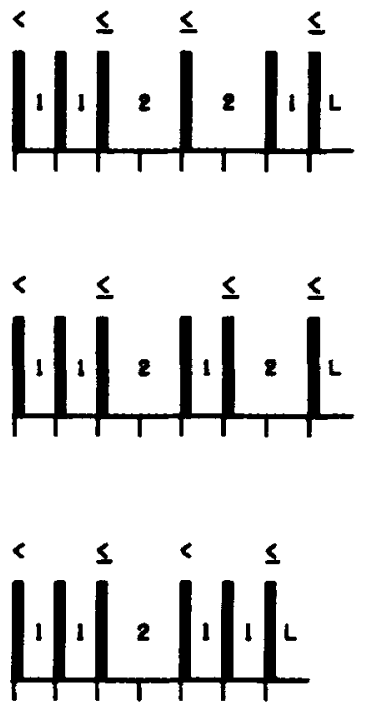

Figure 1. The nine standard 6-tone patterns of Experiment 1. Patterns are shown on an equal-interval timeline and are denoted by the relative sizes of their 5 interonset intervals. Eight patterns comprise 2 long and 3 short intervals. The ninth pattern, 11211, is included as a control. Longs (2s) are twice the length of shorts (1s). A final interval after the sixth tone was reported by subjects to be long. Levels of "natural accenting" are shown for each tone according to the rules of Povel and his colleagues (see text): strong temporal accent is denoted as $\leq$, moderate accent is denoted as <, and the weakest level of accent is not marked. Patterns are arranged in the figure vertically by distance between the 2 long intervals and horizontally by position of the first short interval (see text).

periment 3) and was included here as a control to aid us in comparing results across the two studies. "Long"' (2) is twice the length of "short" ( 1 ).

Figure 1 shows the patterns used in Experiment 1; tone onsets are shown on an equal-interval timeline.

These patterns are not only more complex than isochronic examples, but also contain a different temporal accent structure while comprising (with the exception of the 11211 pattern) the same number and kinds of intervals. We have not recycled our patterns but rather have sampled 8 of 10 possible orders of the total set containing 3 shorts and 2 longs. There are two permutation sets within the total set, each containing five members:

Permutation Set 1: 2211112211112211112221112. Permutation Set 2: 2121112121112122112112112.

The first and second rows of Figure 1 show, respectively, the first three members of the two sets above. The third row of Figure 1 shows the remaining two members of Set $2(21121$ and 12112$)$ as well as the 11211 pattern. In the first row, the 2 longs are adjacent; in the second, they are separated by a short; in the third, they are separated by 2 shorts. The columns in Figure 1 represent the serial position of the first long interval.

Figure 1 also shows the position of the natural temporal accent within each sequence according to Povel and Okkerman (1981) and Povel and Essens (1985). Positions of strong temporal accenting at the beginning of longer intervals are marked with " $\leq$ "; weaker accents for the tone at the end of a long interval that begins the run of 3 or more intervals are marked by " $<$." We consider the last tone in each sequence as accented because it appears to be followed by a long silent interval. Our subjects reported that these patterns seemed to include a final sixth interval which they interpreted as long.

For patterns in the first and third rows, the most prevalent accenting occurs every 2 short (grain) intervals, whereas for patterns in the second row, accenting every 3 grain intervals is just as likely as accenting every 2 grain intervals. The pattern 11211 has the most regular, equally distributed temporal accenting every 2 grain intervals; after an initial short interval, accenting for 12211 and 12112 is also perfectly regular every 2 grain intervals. Accenting in 12121 is regular every 3 grain intervals. With these ensembles, we can study how temporal discrimination for one sound depends upon its position in the pattern-the relation between its position and the organization and length of surrounding intervals. We may also determine whether the chief dependency is on simpler notions of Weberian discrimination or more sophisticated notions of rhythmic simplicity and complexity such as those proposed by Povel and Essens (1985).

\section{Overview of the Design}

Temporal DLs $(\Delta t)$ within patterns of tones were measured at three tempos where the IOIs for short were 50 , 
STRUCTURE OF TRIAL

EXAMPLE: PATTERN 22111 WITH DELAY OF THIRD TONE
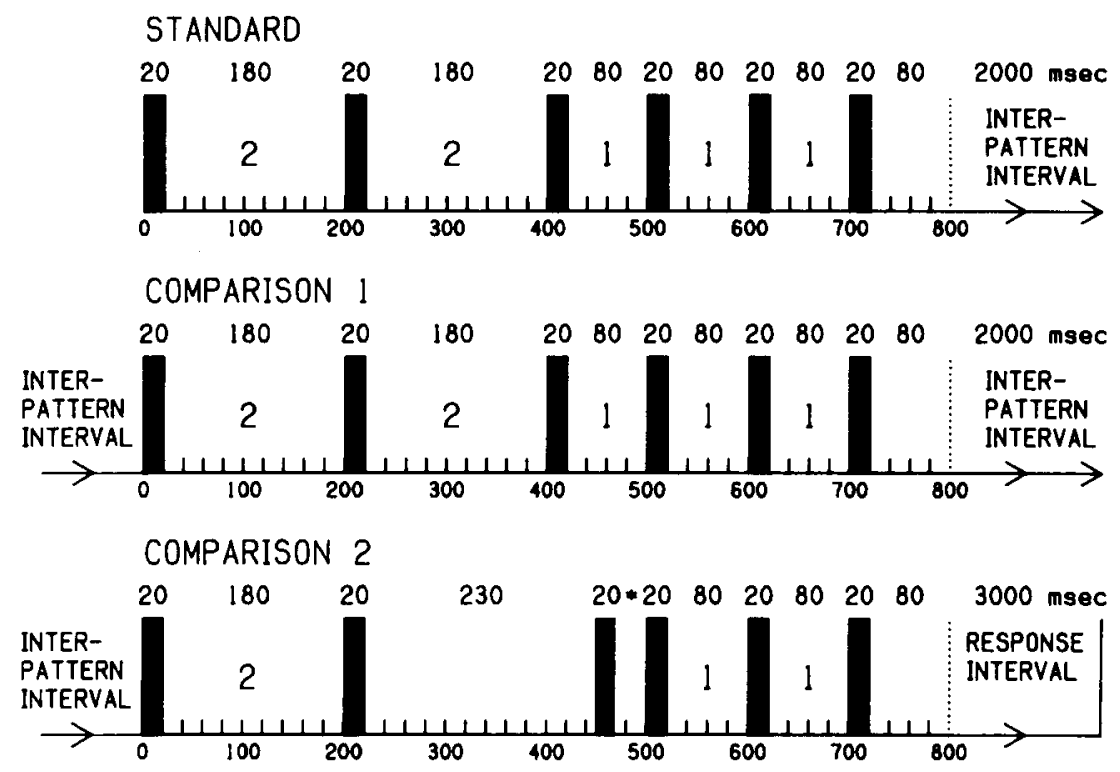

Figure 2. Trial structure with the temporal pattern 22111 as the standard at the medium tempo. Two comparisons follow the standard, one of which contains a delayed tone relative to the tone's position in the standard (the second comparison in the example). Tone durations, silent intervals, interpattern intervals, and response interval are indicated in milliseconds. In Comparison 2, the asterisk (*) represents a 30 -msec silent interval.

100 , or $200 \mathrm{msec}$ and for long were 100,200 , or $400 \mathrm{msec}$, respectively. Tones at Serial Positions 2-6 were delayed for each of the nine patterns. Thus, the experiment may be viewed as a complete factorial comprising 3 tempos $\times 9$ patterns $\times 5$ positions of delay $=135$ conditions.

\section{Method}

Apparatus. A DEC PDP-1173 computer controlled the experiment. Tones were $1000-\mathrm{Hz}$ sinusoids generated from a digital sound file (20,000 samples/sec), passed to a D/A converter, filtered, and sent to earphones in an anechoic room. All tones were $20 \mathrm{msec}$ in length (rise and fall times were $2 \mathrm{msec}$, sustained portion was $16 \mathrm{msec}$ ). Steady-state equivalent sound level at the earphones was maintained at $80 \mathrm{~dB}$ SPL $\pm 0.5 \mathrm{~dB}$ in an artificial ear. Voltage for tones was calibrated daily. The computer collected subject responses and stored them in data files along with stimulus and timing parameters for each trial.

Subjects. There were 4 subjects, 2 males and 2 females, who were paid for their services. All subjects had normal auditory sensitivity. One subject (H.D.) had had 4 years of musical training and had also been a subject in a previous experiment in our laboratory. Another (J.B.) had had 5 years of musical training, was selfrun, and served as experimenter for most of the sessions of the other subjects. The remaining subjects had had 5 and 9 years of musical training, respectively.

Procedure. The subjects were tested singly and, in each replication of the experiment, performed 135 adaptive runs corresponding to the experimental conditions. At the beginning of each run, the experimenter entered the pattern, tempo, and serial position of the tone to be delayed into the program that controlled the adaptive pro- cedure. The experimenter told the subject which tone in the pattern would be delayed. The experimenter played the pattern first in its standard form and then with the initial delay, and ascertained that the subject could hear the difference. The initial delays at the slow, medium, and fast tempos were 50,25 , and $25 \mathrm{msec}$, respectively; these delays had been found to be easily detectable in pilot studies.

The experimenter then initiated the cued $2 \mathrm{AFC}$ procedure in which each trial comprised a standard and two comparison patterns. At random, one of the two comparisons had the delayed tone. Thus, at the middle tempo, the initial trial with the 22111 pattern and delay of the third tone would take the form shown in Figure 2, although the order of the two comparisons might be reversed.

On each trial, the subject indicated which of the two comparisons contained the delay by pushing one of two keys on a computer terminal. The response interval was $3 \mathrm{sec}$; if the subject failed to respond in that time, he or she was prompted to answer by a message on the terminal screen until a response was given. After each response, the terminal screen displayed feedback. In the adaptive procedure, for every two correct answers, the delay was halved; for every incorrect answer, the delay was doubled. For every run, there were five reversals in the amount of detectable delay; each run generally took from 12 to 25 trials. The mean and standard deviation delay detected (in milliseconds) was computed and stored for the last 10 trials of each run. This procedure results in an estimate of $\Delta t$ where the probability of a correct response is $70.7 \%$ (Levitt, 1971).

The subjects replicated the experiment four times, completing one replication before beginning the next. The order of the 135 runs within each replication was randomized across patterns, tempos, and position of delay. Data were analyzed for only the last three replications. Each replication was performed over a period of about 
Table 1

Main Effects and Significant Interactions in Experiment 1

\begin{tabular}{lccc}
\hline \multicolumn{1}{c}{ Effect } & $d f$ & $F$ & $p$ \\
\hline Tempo & 2,6 & 3.33 & .107 \\
Patterns & 8,24 & 2.92 & .020 \\
Position of delayed tone & 4,12 & 4.68 & .017 \\
Replications & 2,6 & 9.74 & .013 \\
Tempo $\times$ patterns & 16,48 & 2.80 & .003 \\
Tempo $\times$ position of delayed tone & 8,24 & 2.92 & .020 \\
Patterns $\times$ position of delayed tone & 32,96 & 6.29 & .0001 \\
Tempo $\times$ patterns $\times$ position of delayed tone & 64,192 & 3.22 & .0001 \\
\hline
\end{tabular}

Table 2

Mean Difference Limens (in msec) for Main Effects of Experiment 1

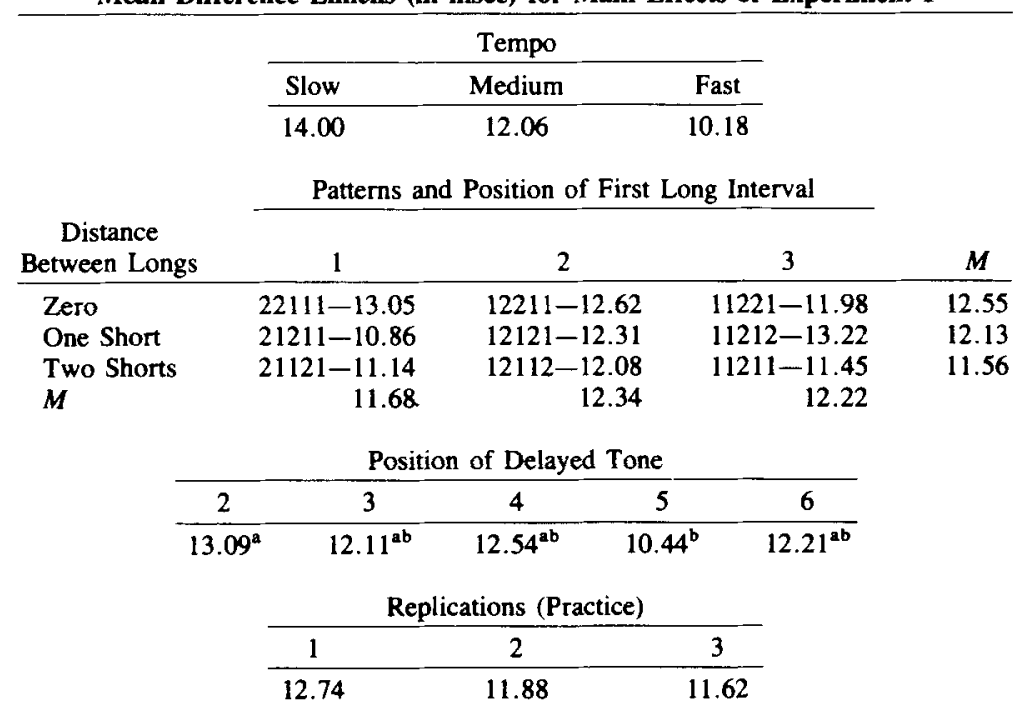

Note-Means with different superscripts differ from each other $(p<.05)$ as measured by Newman-Keuls post hoc tests.

2 weeks with no more than one 2 -h session per day. The listeners typically performed 18 to 20 adaptive runs per 2 -h session. Fiveminute breaks were given after every five adaptive runs.

\section{Results}

A repeated measures analysis of variance (ANOVA) was run on estimates of the absolute DL for delay (in milliseconds) for the 135 experimental conditions. In a complete factorial design, the analysis had 3 tempos $\times 9$ patterns $\times 5$ positions of the delayed tone $\times 3$ replications for each of the 4 subjects. All main effects except tempo were significant, as shown in Table 1.

Main effects. The means for each main effect, as well as levels of main effects that differed significantly from one another, are shown in Table 2. Post hoc analyses were Newman-Keuls tests with alpha level $p<.05$ for the whole experiment.

1. Tempo. Even though the main effect for tempo was not significant, the order of tempo means was the same as that found by Hirsh et al. (1990, Experiment 3) and as that in Experiment 2 (see below). The average absolute DL $(\Delta t)$ was lowest for the fast tempo and highest for the slow tempo.
2. Patterns. The significant effect of pattern seems to be related to the high values of $\Delta t$ in patterns that had 2 adjacent long intervals (patterns in row 1 of Figure 1) and in patterns that had a long interval preceding the final tone (11212 and 12112). None of the differences among patterns was significant by the Newman-Keuls test.

3. Position of delay. Absolute DLs were lowest when Tone 5 was delayed and highest when Tone 2 was delayed (this was the only significant difference that remained so in the Newman-Keuls test); delays of other tones had intermediate effects on DL. This effect may be an artifact, because fewer long intervals abutted Tone 5 than Tone 2 in the different patterns.

4. Practice. Absolute DLs improved (became smaller) with each succeeding replication. The effects of practice did not interact significantly with any other variable.

Interactions. Although the main effects here are of some interest, our original focus is better served by an analysis of the interactions.

First, does discrimination performance depend on the interaction of pattern (i.e., the position of the long intervals) and the position of tone delay? The answer to this question is yes $(p<.0001)$. 
Second, given that there is an interaction between pattern and tone delay, does that interaction depend on the tempo of the patterns? The answer to this question is also yes $(p<.0001)$.

Figure 3 shows the interaction of pattern and position of the delayed tone on the absolute DLs, with tempo shown as the parameter. The pattern is indicated by the length of intervals along the abscissa, so the first pattern in Figure 3 is 22111, and so forth. Each subject's performance was averaged over three replications for each experimental condition. Each point on the graphs of
Figure 3 represents an average of these averages; the standard error for each point is thus based on an $n$ of 4 . Standard errors less than $1 \mathrm{msec}$ are not shown.

At all tempos, DLs tended to be higher when the delayed tone marked the boundary between 2 long intervals (i.e., in Patterns 22111, 12211, and 11221) or when Tone 6 was delayed and was preceded by a long interval (i.e., in Patterns 11212 and 12112). At the slow tempo, DLs were generally smallest (performance was best) when the delayed tone was the boundary between 2 short intervals and were of intermediate value when the delayed tone was
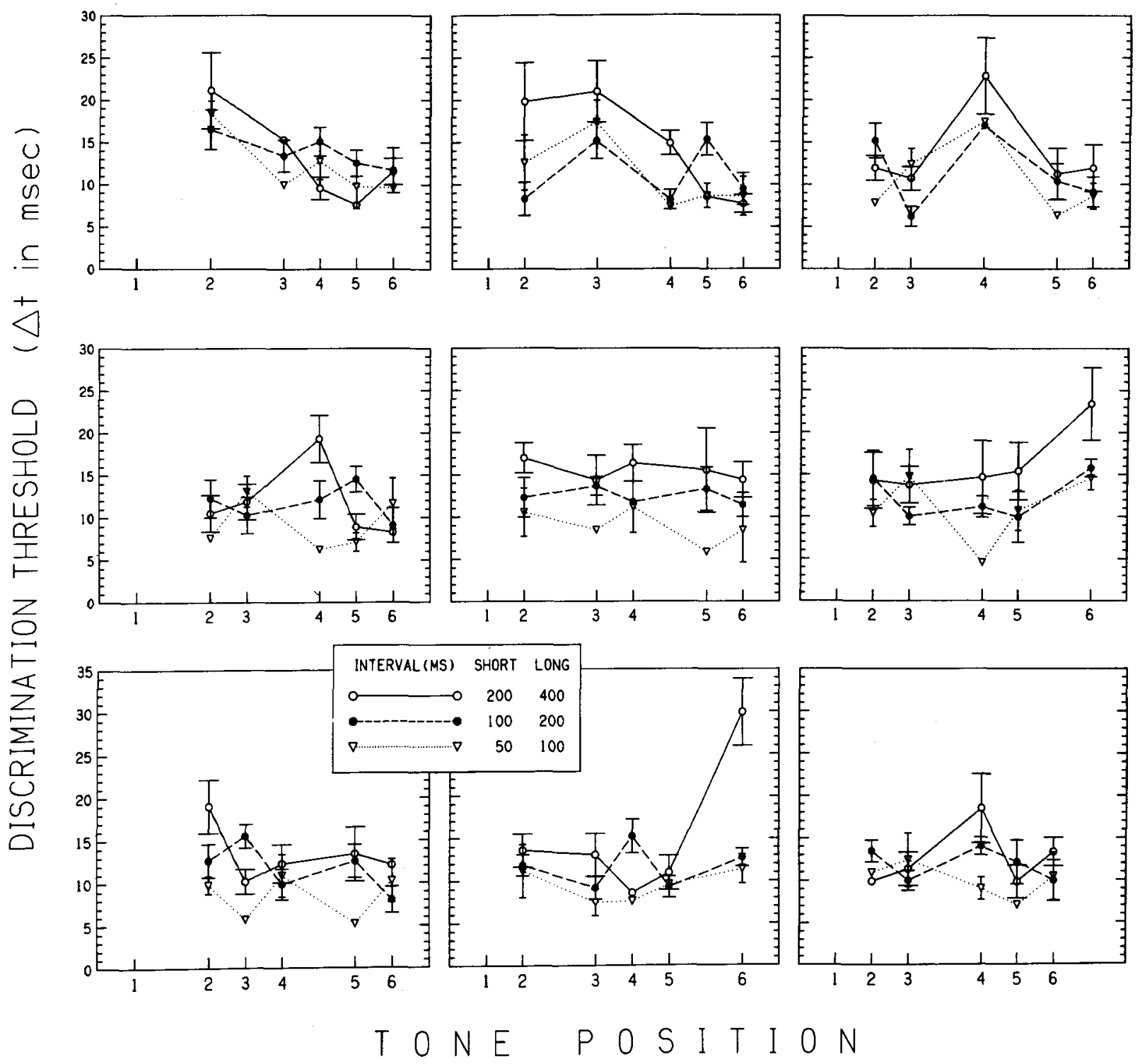

Figure 3. Absolute difference limens (DLs) for delay ( $\Delta t$ in milliseconds) for nine temporal patterns comprising 6 tones and 5 intervals (top row, 22111, 12211, 11221; middile row, 21211, 12121, 11212; bottom row, 21121, 12112, 11211). In each pattern, Tone 2, 3, 4, 5, or 6 was delayed. The absolute DL, measured at each of three tempos, is shown as the parameter. Each point is the average of 4 subjects' average DL across three replications of conditions. Standard errors are based on an $n$ of 4 subjects; standard errors less than 1 msec are not shown. Absolute DLs are related to tempo: the faster the tempo, the lower the absolute DL. 
the boundary between short and long or long and short intervals. This result may be interpreted as following Weber's law, a point to which we shall return.

The results at the medium tempo may not be interpreted as following Weber's law because DLs were almost as large for delays between 2 short intervals (1-1 delays) as they were for delays between 2 long intervals (2-2 delays).

At the fast tempo, absolute DLs were generally higher for tones delayed between a short and a long interval (i.e., for 1-2 delays) than for tones between a long and a short interval (i.e., for 2-1 delays). This asymmetry, which was not present at the slow and medium tempos, contributes greatly to the three-way interaction of tempo, pattern, and delay position.

Finally, we note that performance for the control pattern 11211 in the present experiment was similar to that found by Hirsh et al. (1990) for the same pattern. This pattern is heard as short-short-long-short-short-long; performance at the slow and medium tempos was poor for the fourth tone in the series (ending the first long interval and beginning the following short). This may indicate a grouping effect where listeners are quite accurate in hearing timing differences within temporal groups but not between groups.

We defer further discussion of the results of Experiment 1 -in particular, measures of relative $D L-$ and Weberian or accent models of discriminability until after the results of Experiment 2 have been presented.

\section{EXPERIMENT 2}

\section{Patterns}

The nine patterns of Experiment 2 were mirror images of those of Experiment 1 in that long intervals were substituted for short, and vice versa. Thus, the patterns had 2 short and 3 long intervals, with the exception of the 22122 pattern, which is the mirror image of the 11211 pattern of Experiment 1. Figure 4 shows these patterns along with positions of temporal accent as suggested by Povel and his colleagues. All patterns in the first row of Figure 4 have regular temporal accenting every 2 grain intervals. Patterns in rows 2 and 3 have more irregular patterns of accenting; accenting every 3 grain intervals is most prevalent for the former, and accenting every 2 grain intervals is most prevalent for the latter. In addition, the 21212 pattern may be heard as a repeated long-short pattern with regular accenting (every 3 grain intervals), and the 21221 pattern tends to be heard as a repeated long-short-long rhythm, since the interval after the final tone of the pattern is usually heard as long.

\section{Overview of the Design}

The tempos and positions of tone delay employed in the present experiment were the same as those employed in Experiment 1. Thus, the designs for the two experiments, except for the use of nine different temporal patterns, were identical-namely, complete factorials with

\section{POSITION OF FIRST SHORT INTERVAL}

\section{DISTANCE BETUEEN} -SHORTS"

ZERO "LONGS"
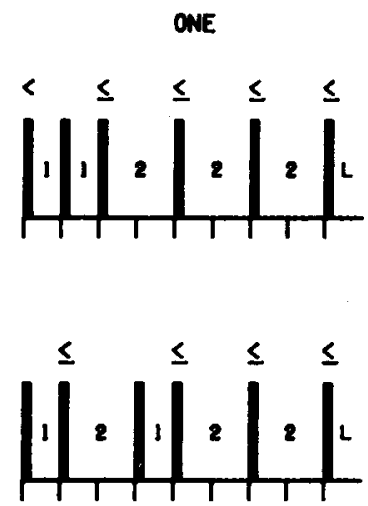
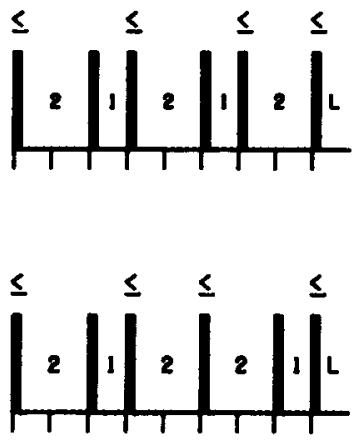

THREE
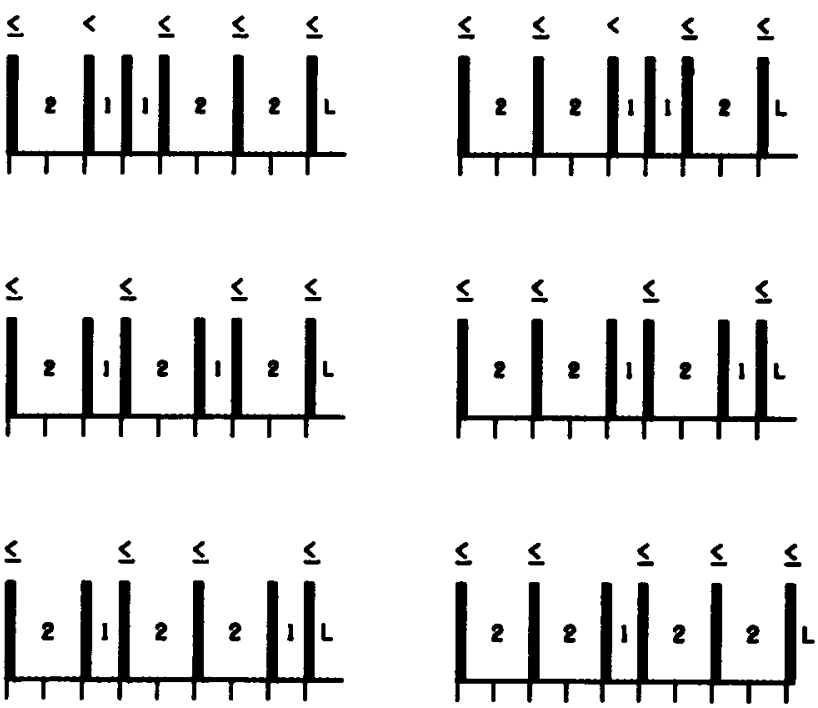

Figure 4. The nine standard 6-tone patterns of Experiment 2. Patterns are mirror-images of those for Experiment 1, so that short intervals are now in the positions previously held by long intervals, and vice versa. Thus, eight of the patterns have 3 long and 2 short intervals and Pattern 22122 is the mirror of Pattern 11211. The levels of natural accent for each tone are shown according to the rules of Povel and his colleagues (see text). Patterns are arranged in the figure vertically by the distance between the 2 shorts and horizontally by the position of the first short interval. 
3 tempos $\times 9$ patterns $\times 5$ positions of delay, totaling 135 conditions.

\section{Method}

There were 3 subjects, 1 male and 2 females, with 4, 5, and 10 years of musical experience, respectively. All subjects had normal auditory sensitivity. Two of the subjects, J.B. and H.D., had also been subjects in Experiment 1. J.K., the new listener, like J.B., was a self-run subject. The subjects were paid for their services.

Both the apparatus and the procedure were the same as in Experiment 1.

\section{Results}

A repeated measures ANOVA was run on estimates of the absolute DL for delay in milliseconds for the $135 \mathrm{ex}-$ perimental conditions. In a complete factorial design, the analysis had 3 tempos $\times 9$ patterns $\times 5$ positions of the delayed tone $\times 3$ replications for each of the 3 subjects. Table 3 shows the main effects and significant interactions of this analysis. Table 4 shows the average for each level of each main effect for the experiment.

Main effects. In this experiment, tempo was the only main effect that was significant. The smallest absolute DLs occurred for the fast tempo, and the largest occurred for the slow tempo. The same order of tempo means was found in Experiment 1 (compare Tables 2 and 4 for average absolute DLs for each tempo). Hirsh et al.'s (1990) Experiments 1 and 3 also had the same order of average absolute DLs. This very consistent result implies that Weber's law does not hold across tempos in the range we are examining. If Weber's law were in effect for this range, then the average $\Delta t$ for the slow-tempo condition should be twice that of the medium-tempo condition, which, in turn, should be twice that of the fast-tempo condition. This is clearly not the case.

We note that although the main effect for patterns was not significant, patterns in the first row of Figure 4 that had 2 adjacent shorts or had regular accenting every 2 grain intervals averaged a somewhat lower DL than did patterns in the other rows (see levels of main effects for patterns in Table 4).

Interactions. The same two interactions that were most highly significant in the first experiment-namely, the two-way interaction of pattern (the position of the various intervals) with the position of delay, and the three-

Table 3

Main Effects and Significant Interactions in Experiment 2

\begin{tabular}{llcc}
\hline \multicolumn{1}{c}{ Effect } & $d f$ & $F$ & $p$ \\
\hline Tempo & 2,4 & 20.89 & .008 \\
Patterns & 8,16 & 1.42 & .260 \\
Position of delayed tone & 4,8 & 1.97 & .193 \\
Replications & 2,4 & 0.08 & .925 \\
Patterns $\times$ position of delayed tone & 32,64 & 4.68 & .0001 \\
Tempo $\times$ patterns $\times$ position of delayed tone & 64,128 & 2.19 & .0001 \\
\hline
\end{tabular}

Table 4

Mean Difference Limens (in msec) for Main Effects of Experiment 2

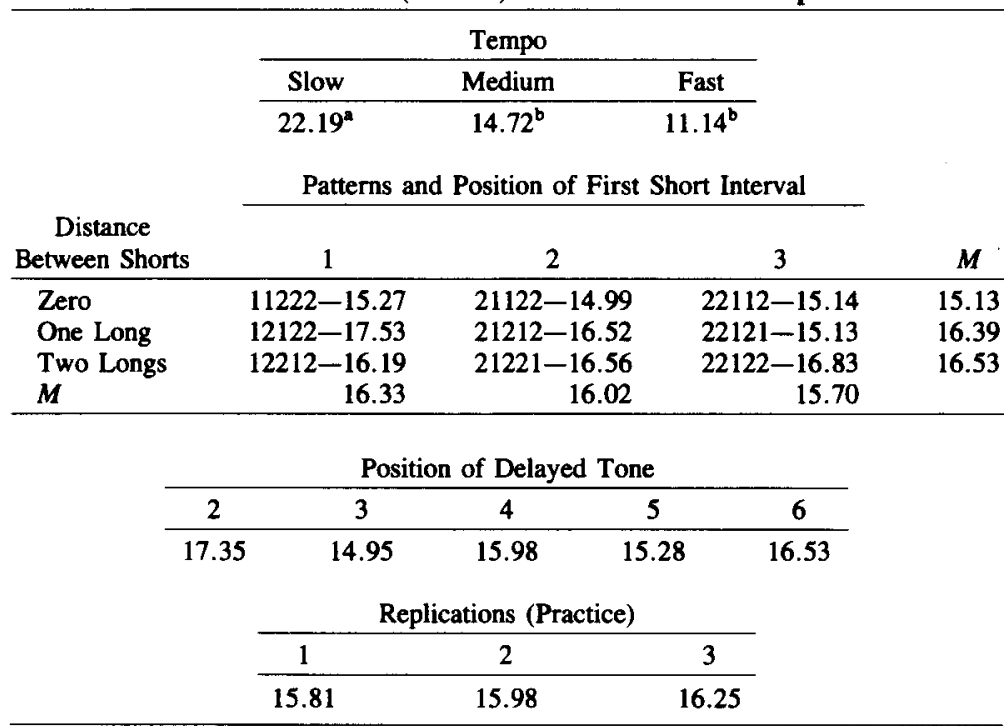

Note-Means with different superscripts differ from each other $(p<.05)$ as measured by Newman-Keuls post hoc tests. 

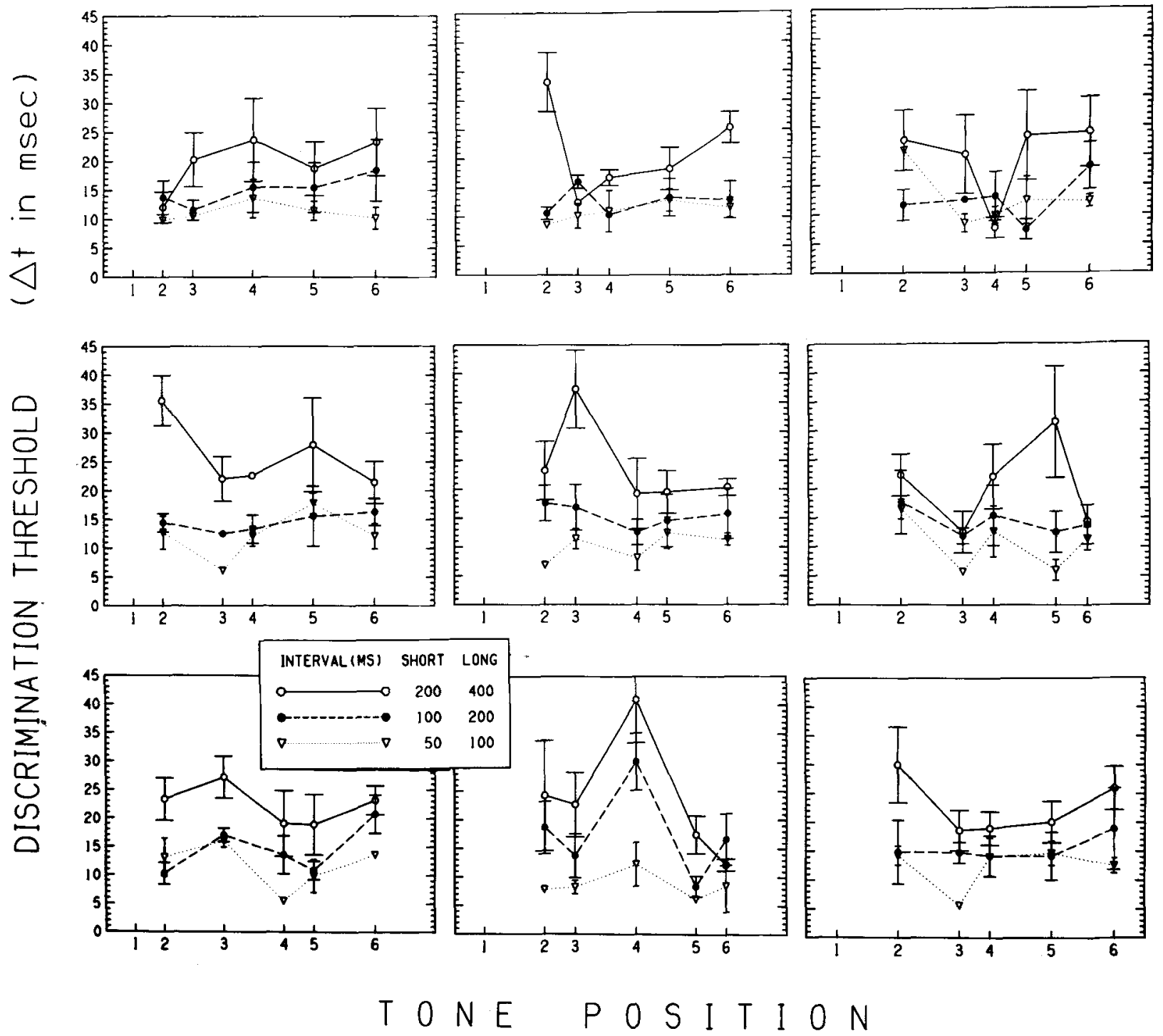

Figure 5. Absolute difference limens (DLs) for delay ( $\Delta t$ in milliseconds) for nine temporal patterns comprising 6 tones and 5 intervals (top row, 11222, 21122, 22112; middle row, 12122, 21212, 22121; bottom row, 12212, 21221, 22122). In each pattern, Tone 2, 3, 4, 5, or 6 was delayed. The absolute DL, measured at each of three tempos, is shown as the parameter. Each point is the average of 3 subjects' average DL across three replications of conditions. Standard errors are based on an $n$ of 3 subjects; standard errors less than 1 msec are not shown. Absolute DLs are related to tempo: the faster the tempo, the lower the absolute DL.

way interaction of tempo with pattern and position of delay on the absolute DL-were also highly significant in Experiment 2 (compare interactions in Tables 1 and 3).

Figure 5 shows the interaction of pattern and position of the delayed tone on the absolute DLs, with tempo shown as the parameter. The pattern is indicated by the length of intervals along the abscissa, so the first pattern in Figure 5 is 11222, and so forth. Each subject's performance was averaged over three replications for each experimental condition. Each point on the graphs of Figure 5 represents an average of these averages; the standard error for each point is thus based on an $n$ of 3 . Standard errors less than $1 \mathrm{msec}$ are not shown.
As in the first experiment, delays between 2 longs yielded a high $\Delta t$. At the slow tempo, delays of Tone 6 were much easier to detect if they were preceded by a short interval (e.g., in Patterns 22121 and 21221) than if they were preceded by a long interval. Also, at the slow tempo, $\Delta t$ was smallest for delays between 2 shorts; these outcomes, as noted above, are in correspondence with Weber's law.

At the medium tempo, delays between 2 shorts were poorly detected-as was the case in the first study. At the fast tempo, we again found a large disparity in sensitivity to 1-2 and 2-1 delays, with the latter being far better detected than the former. 

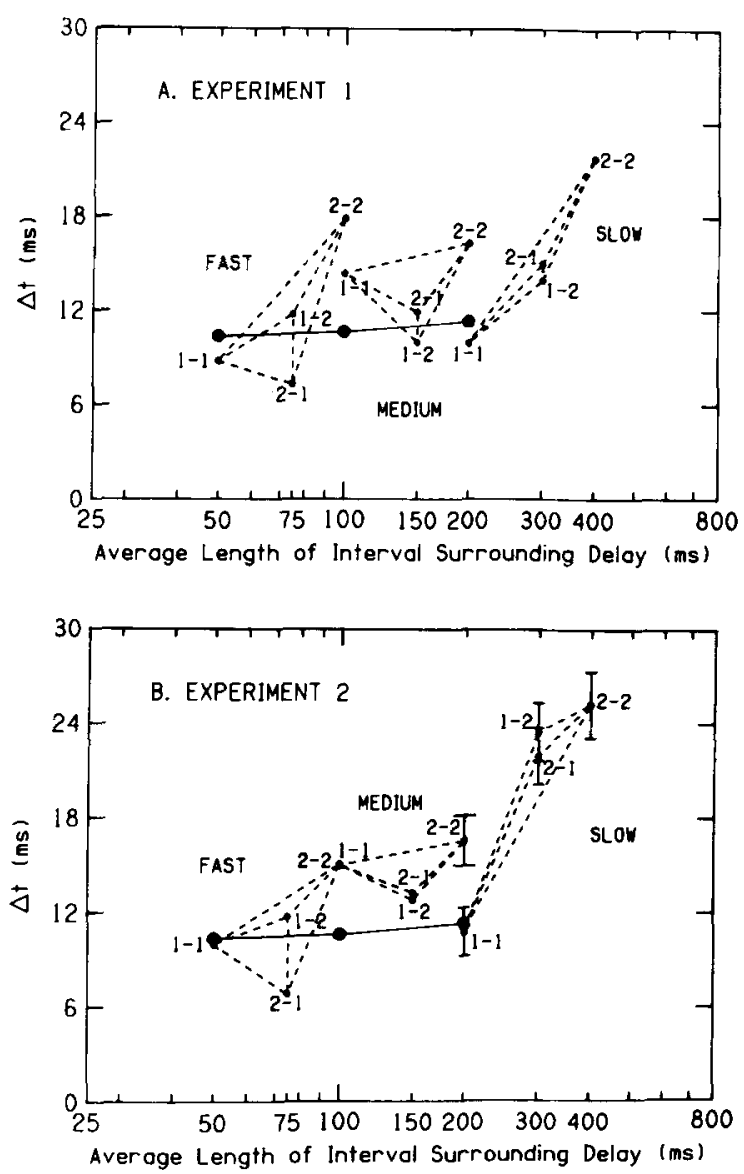

Figure 6. The value of the absolute difference limen $(\mathrm{DL} ; \Delta t)$ at three different tempos and in four delay contexts $(1-1,1-2,2-1$, and 2-2; see text) for Experiments 1 (Panel A) and 2 (Panel B). Standard errors for each point in the figures are based on the number of occurrences of the four contexts in each experiment (see text); standard errors less than $1 \mathrm{msec}$ are not shown. Contexts within the same tempo are connected by dashed lines. The abscissa is the average size of the 2 intervals within which the delayed tone occurred. An additional reference (solid line) is provided, showing the absolute DL for the delay of a single tone in otherwise isochronic sequences (Hirsh et al., 1990, Experiment 1), where isochronic intervals were 200,100 , or 50 msec.

Another way of viewing the results. From the above noted correspondences, it seemed likely that the results of the two experiments would be quite similar if seen as a function of delay context-that is, the intervals surrounding the delayed tone-and tempo.

Figures $6 \mathrm{~A}$ and $6 \mathrm{~B}$ show the value of the absolute DL at the three different tempos in the four delay contexts (1-1, 1-2, 2-1, 2-2) in Experiments 1 and 2. The two experiments have different numbers of these contexts. Excluding delays of Tone 6 , there are 36 delay contexts in each experiment divided among the four types as indicated in Table 5. Standard errors for each point in Figure 6 are based on the number of contexts for each delay type; standard errors less than $1 \mathrm{msec}$ are not shown. Contexts within the same tempo are connected by dashed lines. The abscissa in these figures is the average size of the 2 intervals within which the delayed tone occurred. Thus, the average interval size surrounding a delay for both 1-2 and 2-1 delays is 300,150 , or $75 \mathrm{msec}$ for slow, medium, and fast tempos, respectively. However, a 1-2 delay context means that the order of intervals in the standard pattern was 200-400,100-200, or 50-100 msec, respectively, whereas a 2-1 delay context means the reverse order of intervals. An additional reference (solid line) shows the absolute DL $(\Delta t)$ for the delay of a single tone in otherwise isochronic sequences (Hirsh et al., 1990, Experiment 1) where isochronic intervals were 200,100 , or $50 \mathrm{msec}$.

To estimate the similarity in outcomes of Experiments 1 and 2, we correlated the average absolute DLs for the 12 contexts in Figure 6A (4 delay contexts at each of 3 tempos for Experiment 1) with the same 12 contexts for Experiment 2 in Figure 6B, and found $r(10)=.804$ $(d f=10, p \leq .001)$.

At all tempos in both experiments, delays between 2 longs (2-2 delays) gave the highest average DLs (see Figures 6A and 6B). At the slow tempo, $\Delta t$ increased with the average size of the intervals surrounding the delay. At the medium and fast tempos, this was not the case. The result within slow-tempo patterns is consistent with Weber's law. We also note that our slow-tempo results agree with the findings of Bharucha and Pryor (1986), who employed a different procedure for investigating the discriminability of timing. They increased the length of single intervals by half a beat (and thereby increased total pattern length) in rhythmic patterns that were made up of relatively random sequences of intervals. They found that as $t$ (the size of the interval) increased, $\Delta t$ (the absolute $\mathrm{DL}$ ) also increased. The size of temporal intervals in their experiment ranged from 200 to $1,200 \mathrm{msec}$. However, the absolute DLs for 1-2 and 2-1 delays (average interval $=300 \mathrm{msec}$ ) at the slow tempo were 7-9 $\mathrm{msec}$ higher in our Experiment 2 than in Experiment 1; at present, we have no good explanation for this result.

The experiments agree fairly well in levels of the absolute DL at medium and fast tempos, especially in the unpredicted result that 2-1 delays are much better detected at the fast tempo than are 1-2 delays.

The experiments agree on a further interesting outcome. The delay between 2 shorts at the slow tempo is physically equal, in terms of surrounding intervals, to the delay between 2 longs at the medium tempo. The difference in $\Delta t$ to the same physical delay in the two different tempo contexts in both experiments is slightly above $6 \mathrm{msec}$. The

Table 5

Distribution of Delay Contexts

\begin{tabular}{llcc}
\hline & & \multicolumn{2}{c}{$n$ s } \\
\cline { 3 - 4 } \multicolumn{1}{c}{ Delay Context } & & Experiment 1 & Experiment 2 \\
\hline Between two shorts & $(1-1)$ & 10 & 3 \\
Between long and short & $(1-2)$ & 11 & 12 \\
Between short and long & $(2-1)$ & 12 & 11 \\
Between two longs & $(2-2)$ & 3 & 10 \\
\hline
\end{tabular}


standard context for these delays contains 2 consecutive 200 -sec intervals. However, at the medium tempo, the delay sounds as if it occurs between temporal groupings (between 2 longs), whereas at the slow tempo, the delay sounds as if it occurs within a temporal grouping (between 2 shorts).

\section{DISCUSSION}

\section{A Weber Fixed-Beat Model}

One reasonable choice for a reference standard in the Weber ratio is $t=$ length of the short or long interval (the most prevalent interval). If the Weber ratio were constant across tempos, then the values at each of the three tempos should have the same vertical position in Figures 7A and $7 \mathrm{~B}$, which show relative sensitivity ( $\Delta t / t$ short and $\Delta t / t$ long) for delay in four different contexts in the two dif-
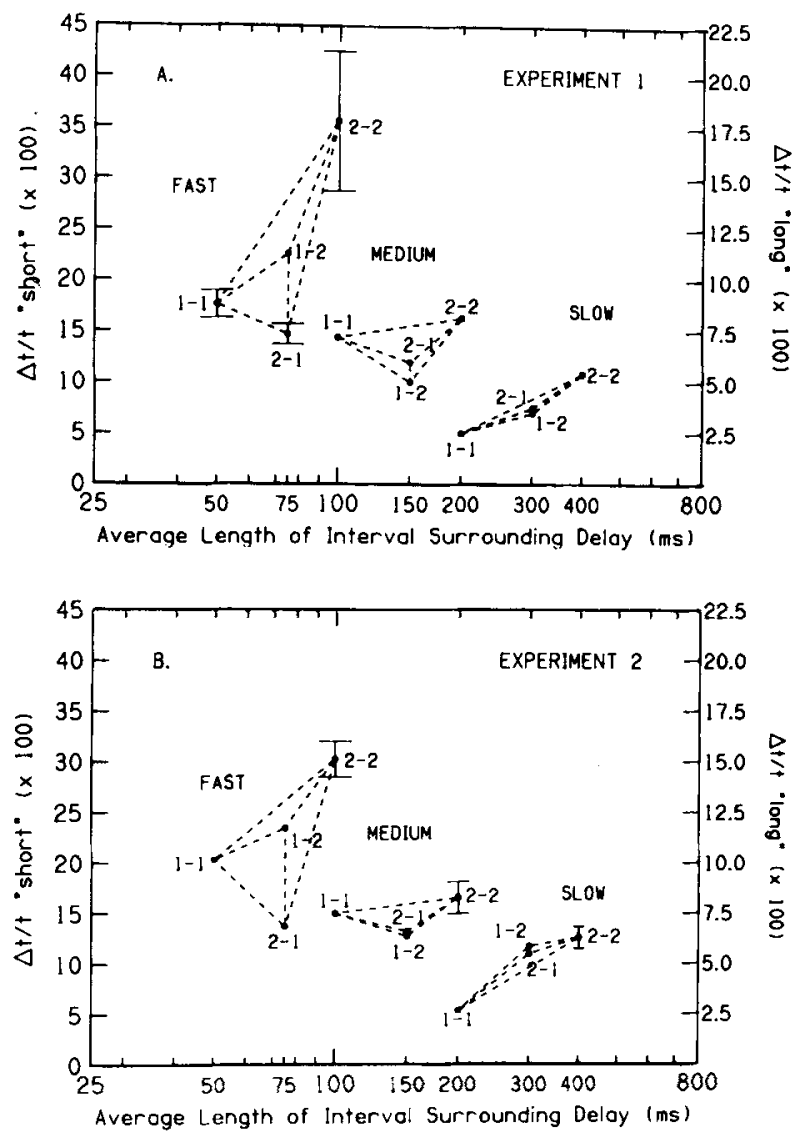

Figure 7. Value of the relative difference limen (the Weber ratio = $\Delta t / t$ "short" or $\Delta t / t$ "long," left- and right-hand vertical scales, respectively, in each panel) at three tempos and in four delay contexts (1-1, 1-2, 2-1, and 2-2; see text) for Experiments 1 (Panel A) and 2 (Panel B). Standard errors for each point in the figures are based on the number of occurrences of the four contexts in each experiment (see text); standard errors less than $1 \Delta t / t$ unit are not shown. Contexts within the same tempo are connected by dashed lines. The abscissa is the average size of the 2 intervals within which the delayed tone occurred. ferent experiments, respectively. We are investigating here the boundary where Weber's ratio for discrimination of time begins to be constant for the comparison of single intervals, apparently somewhere between 100 and $200 \mathrm{msec}$ (see Killeen \& Weiss, 1987, for a recent review). In fact, $\Delta t / t$ short or long increases with increasing tempo or decreasing size of either the shorter or the longer interval; this is in general agreement with the literature for judgments of the lengths of single intervals, which shows that the Weber ratio increases monotonically and steeply with decreasing interval lengths under $100 \mathrm{msec}$.

Using a constant value for $t$ at each tempo carries the assumption that a clock-generated beat or pulse is used for making discriminations, whether a tone is present marking the onset of that beat interval or not. However, the implementation of this model, especially at the slow tempo, implies that the delays of isolated tones, tones followed, and in some cases preceded, by long intervals ("accented" tones in Povel \& Essens's, 1985, terms), have higher relative thresholds for temporal discrimination (see slow-tempo values in Figures 7A and 7B). On the other hand, on the basis of research with comparison of single temporal intervals, we might expect a constant Weber ratio within the slow tempo. Both Getty (1975) and Kristofferson (1980) have reported a constant ratio between .05 and .06 for single intervals of 200 and $400 \mathrm{msec}$, which are, respectively, the short and long of our slow-tempo patterns.

\section{A Weber Average-Interval Model}

Another version of the Weber hypothesis assumes that the listener employs both the preceding and the following intervals in making a discrimination. Thus, the $t$ in the denominator of the Weber ratio is the average of the 2 intervals separated by the delayed tone.

Figures $8 \mathrm{~A}$ and $8 \mathrm{~B}$ show the Weber ratio calculated on an averaged $t$ for each of the four delay contexts at each of the three tempos for Experiments 1 and 2. The fact that data for the four contexts at the slow tempo fall in a horizontal straight line, especially for Experiment 1 (see Figure 8A), suggests that this type of model will provide a good fit. In both Figure 8A and Figure 8B, the distribution of the 12 points for $\Delta t / t$ average interval forms a curve that is quite reminiscent of that for $\Delta t / t$ for single intervals, with two possible exceptions, for which we can present only ad hoc explanations.

First, in both experiments, according to the $\Delta t / t$ average measure, delays were much better discriminated between 2 shorts at the slow tempo than between 2 longs at the medium tempo, despite the fact that both delays had the same sizes of surrounding interval. We suggested above that this result had to do with whether the intervals in question were within-groups temporal intervals or between-groups intervals. Povel (1981) has observed a perceptual difference between the two types of interval. In his study, listeners reproduced sequences with alternating short and long intervals, which he called $t_{1}$ and $t_{2}$, respectively. He states: 
If $a t_{1}-t_{2}$ pattern is presented to a subject, beeps and not intervals will be perceptually grouped according to their proximity. Thus $t_{1}$ becomes part of the group (figure) and $t_{2}$ part of the background.... Between-groups intervals are special. Subjects indicate that such intervals have hardly any reality for them. Indeed, in imitating these sequences one senses that much attention is directed toward imitating the withingroup interval, whereas the between-groups interval hardly requires any attention. (p. 10)

Thus, it is possible that, because of the different attentional demands of the two contexts, our listeners found it easier to hear the same delays in a within-groups context (between 2 shorts at the slow tempo) than in a between-groups context (between 2 longs at the medium tempo).

Second, at the fast tempo, in both experiments, 2-1 delays were much easier to detect than were 1-2 delays. One possible explanation is that the delayed tone is closer to the preceding tone in the 1-2 context and is therefore more subject to forward masking, which is generally sup-
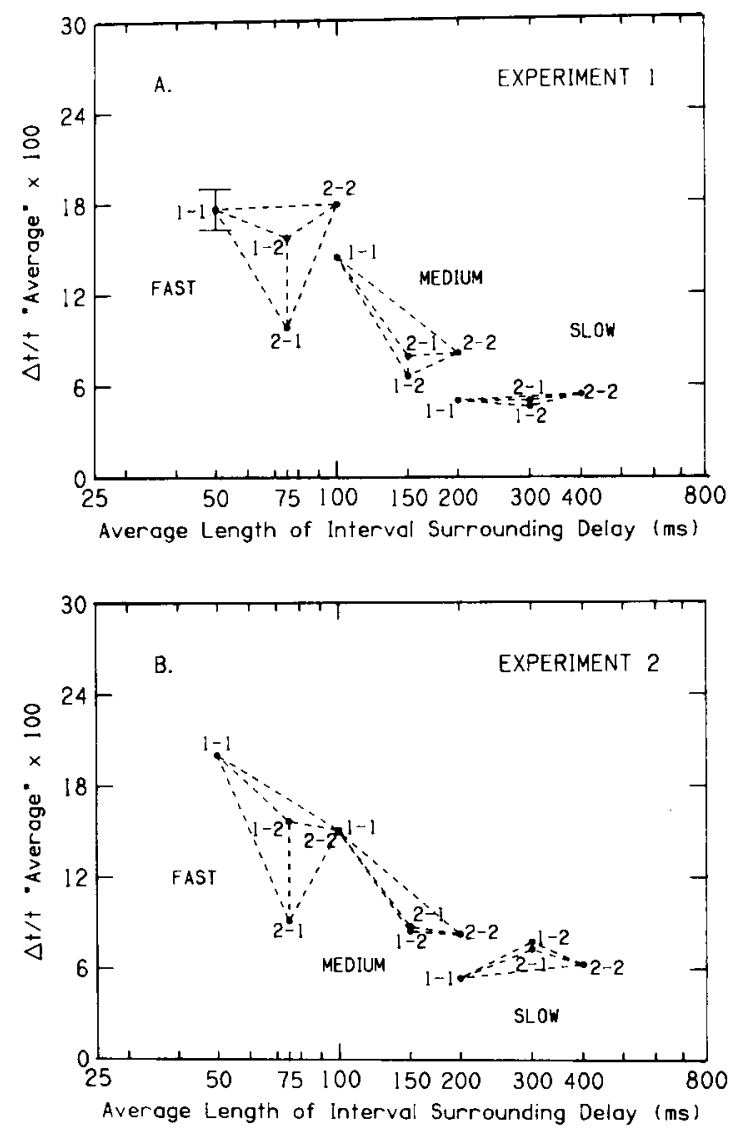

Figure 8. Value of the relative difference limen (the Weber ratio $=$ $\Delta t / t$ "average") at three tempos and in four delay contexts $(1-1,1-2$, 2-1, and 2-2; see text) for Experiments 1 (Panel A) and 2 (Panel B). Standard errors for each point in the figures are based on the number of occurrences of the four contexts in each experiment (see text); standard errors less than $1 \Delta t / t$ average unit are not shown. Contexts within the same tempo are connected by dashed lines. The abscissa is the average size of the 2 intervals within which the delayed tone occurred.
Table 6

Intervals Surrounding the Position of the Delayed Tone

\begin{tabular}{|c|c|c|c|c|}
\hline \multirow{2}{*}{$\begin{array}{l}\text { Delay } \\
\text { Type }\end{array}$} & \multicolumn{2}{|c|}{ Standard } & \multicolumn{2}{|c|}{$\begin{array}{l}\text { Initial Different } \\
\text { Comparison }\end{array}$} \\
\hline & Period (msec) & Rate $(\mathrm{Hz})$ & Period (msec) & Rate $(\mathrm{Hz})$ \\
\hline $1-1$ & $50-50$ & $20-20$ & $75-25$ & $13.3-40$ \\
\hline $1-2$ & $50-100$ & $20-10$ & $75-75$ & $13.3-13.3$ \\
\hline $2-1$ & $100-50$ & $10-20$ & $125-25$ & $8-40$ \\
\hline $2-2$ & $100-100$ & $10-10$ & $125-75$ & $8-13.3$ \\
\hline
\end{tabular}

posed to be stronger than backward masking (Elliott, 1962). However, this result is correlated with a perceptual phenomenon of an apparent pitch and/or timbral change that seems to accompany 2-1 delays but not 1-2 delays. At the fast tempo, the standards for trials with 2-2 delays have a repetition rate of 10 notes $/ \mathrm{sec}$, or $10 \mathrm{~Hz}$, surrounding the position of the tone to be delayed; standards for trials with 1-1 delays have a repetition rate of $20 \mathrm{~Hz}$ surrounding the position of the tone to be delayed. In Table 6, we show the standard and initial changed comparison intervals for the four types of delay context at the fast tempo; the intervals are shown both in terms of their periods (IOIs in milliseconds) and in terms of their repetition rates $(\mathrm{Hz})$. The initial delay in the changed comparison at the beginning of any adaptive run is $25 \mathrm{msec}$. Listeners easily notice the large difference in the repetition rate for $2-1$ contexts and, to a lesser degree, for 1-1 contexts. The different comparison for 1-2 contexts initially exhibits no difference at all in repetition rate. It is possible that we have tapped into what Bharucha and Pryor (1986) have termed an "asymmetry in the detection of alterations to a sequence, as a function of whether coherence is violated or established" (p. 137). Thus, it may be argued that the initial different comparisons for the 2-1 and 1-1 contexts at the fast tempo are more "incoherent" than their respective standards, and hence are easy to detect; on the other hand, the initial delayed comparison for a 1-2 context is just as "coherent" as the standard, because the intervals surrounding the delayed tone are equal in length and repetition rate.

\section{An Accent Model Compared with a Weber's Law Model}

The smallest IOIs for which Povel and Okkerman (1981) report natural temporal accent are about $100 \mathrm{msec}$; thus, it is probably inappropriate to apply a temporal accent model to our fast-tempo patterns. It is also clear from the preceding section that a Weber's law model is not likely to fit data from within patterns at either the fast or the medium tempo. Therefore, we will compare the two models only at the slow tempo as predictors of the 36 absolute DLs we obtained in each experiment ( 9 patterns $\times$ Delay Positions 2, 3, 4, and 5); we will then compare the two models for the 72 DLs from the combined experiments.

Table 7 shows the predictions of the two different models for the discriminability of delay for each of four tone positions $(2,3,4$, and 5) for each pattern in Experi- 
Table 7

Model Weights Predicting $\Delta t$ for Four Positions of Tone Delay (Tones 2, 3, 4, and 5) in 18 Patterns from the Two Experiments

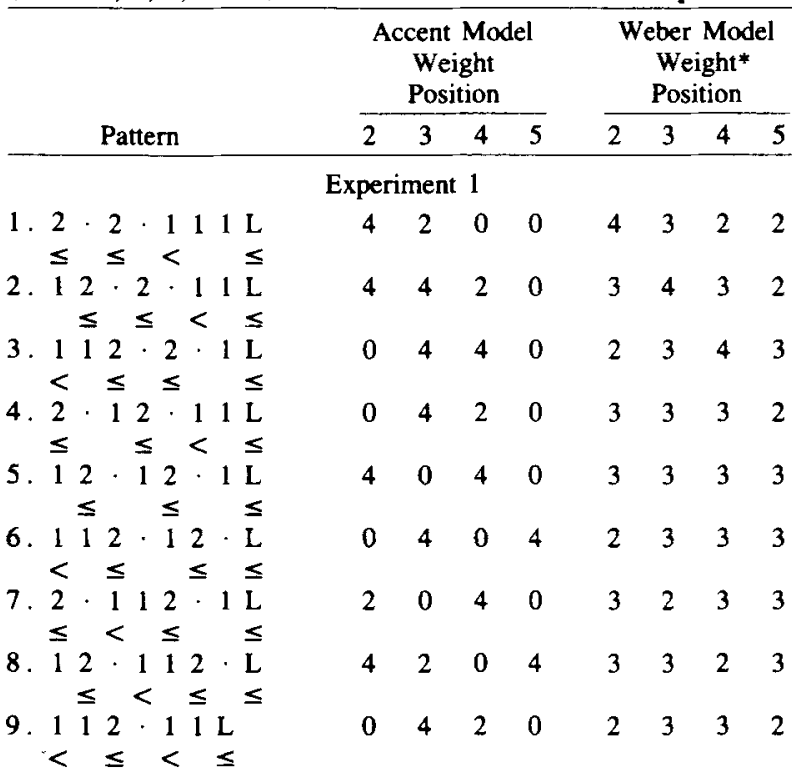

Experiment 2

1. $112 \cdot 2 \cdot 2 \cdot \mathrm{L}$ $<\leq \leq \leq \leq$

$2 \cdot 2 \cdot 1+2 \cdot 2 \cdot L$ $\leq<\leq \leq \leq$

3. $2 \cdot 2 \cdot 112 \cdot \mathrm{L}$ $\leq \leq<\leq \leq$

4. $12 \cdot 12 \cdot 2 \cdot \mathrm{L}$ $\leq \leq \leq \leq$

$5.2 \cdot 12 \cdot 12 \cdot \mathrm{L}$

$\leq \leq \leq \leq$

6. $2 \cdot 2 \cdot 12 \cdot 1 \mathrm{~L}$ $\leq \leq \leq \leq$

7. $12 \cdot 2 \cdot 12 \cdot \mathrm{L}$ $\leq \leq \leq \leq$

8. $2 \cdot 12 \cdot 2 \cdot 1 \mathrm{~L}$

$\leq \leq \leq \leq$

9. $2 \cdot 2 \cdot 12 \cdot 2 \cdot \mathrm{L}$ $\leq \leq \leq \leq \leq$

$\begin{array}{llllllll}0 & 4 & 4 & 4 & 2 & 3 & 4 & 4 \\ 2 & 0 & 4 & 4 & 3 & 2 & 3 & 4 \\ 4 & 2 & 0 & 4 & 4 & 3 & 2 & 3 \\ 4 & 0 & 4 & 4 & 3 & 3 & 3 & 4 \\ 0 & 4 & 0 & 4 & 3 & 3 & 3 & 3 \\ 4 & 0 & 4 & 0 & 4 & 3 & 3 & 3 \\ 4 & 4 & 0 & 4 & 3 & 4 & 3 & 3 \\ 0 & 4 & 4 & 0 & 3 & 4 & 3 & 3 \\ 4 & 0 & 4 & 4 & 4 & 3 & 3 & 4\end{array}$

Note-Patterns are shown with levels of natural accent. $\leq=$ strong temporal accent. < = moderate temporal accent. *Weber model weights are the mean of 2 intervals around the delayed tone divided by 100 .

ments 1 and 2 . The accent model that we test here predicts that temporal sensitivity is inversely related to the level of perceived temporal accent as described by Povel and Okkerman (1981) and Povel and Essens (1985). The model provides an ordinal scale of three levels of temporal accent: (1) Delays of tones that initiate long intervals, marked " $\leq$ " in Table 7 (the markings are identical to those in Figures 1 and 4), will be poorly detected ( $\Delta t$ will be large); (2) delays of tones that begin a series of 3 or more intervals, marked " < " in Table 7, will be moderately well detected; and (3) delays of tones that are unmarked by temporal accent will be best detected ( $\Delta t$ will be small). The initial weights of the accent model were not chosen entirely arbitrarily. Povel and Okkerman
(Experiment 4) played repeating sequences consisting of alternating long and short intervals. As noted above, the tones that are followed by the long interval in such a series sound accented. In their Experiment 4, Povel and Okkerman had their subjects raise the level of the unaccented tones in the series (tones followed by short intervals) until they sounded as loud and important as the temporally accented tones. In general, the subjects had to raise the level by about $4 \mathrm{~dB}$. Thus, we assume that the relative levels of accent for strongly accented and unaccented tones in the model differ by $4 \mathrm{~dB}$. We have arbitrarily chosen 0 and $4 \mathrm{~dB}$ as low and high values of accent, and employed $2 \mathrm{~dB}$ as the middle value, which is evenly interpolated between 0 and 4 .

The Weber averaged-interval model, on the other hand, predicts that $\Delta t$ is proportional to the average of the lengths of the 2 intervals surrounding the delayed tone. The slowtempo average interval for $1-1$ delays is $200 \mathrm{msec}$, for 1-2 or 2-1 delays is $300 \mathrm{msec}$, and for 2-2 delays is $400 \mathrm{msec}$. These average values, divided by 100 , give the weights for the Weber model shown in Table 7.

Three types of correlation are shown in the top portion of Table 8: (1) Model weights are correlated with each other for each experiment and for both the accent and the Weber models; (2) model weights for both models are correlated with experimental results $(\Delta t)$ for the 36 conditions of Experiment 1 and the 36 conditions of Experiment 2; and (3) $\Delta t$ for the 36 conditions of Experiment 1 is correlated with $\Delta t$ for the 36 conditions of Experiment 2.

As can be seen from the table, the Weber averagedinterval model predicts $\Delta t$ better for both experiments than does the accent model, and, furthermore, it accounts for more than half the variance $\left(R^{2}=.594\right)$ of the 36 points from Experiment 1 . The Weber model, without involving the estimation of any parameters at all, does a good job of fitting the data from Experiment 1 and a mediocre job of fitting the data of Experiment 2. The accent model fits Experiment 1 data slightly better than it does those of Experiment 2, but the fit to both data sets is poor at best.

We note that the predictions of the two models are significantly correlated $(r=.642)$ across the the 72 conditions of the two experiments. The partial correlation of the Weber model predictions with $\Delta t$ from the 72 conditions from both experiments-holding the predictions of the accent model constant-is $.529(d f=70, p<.001)$. On the other hand, the partial correlation of the predictions of the accent model with $\Delta t$ from the same 72 conditions from the two experiments-holding predictions of the Weber model constant-is $.07(d f=70, p=n . s$. $)$. Thus, we may conclude that the accent model predicts very little of the variance in discrimination behavior independently from what is predicted by the Weber model.

\section{SUMMARY}

We have found that trained listeners can discriminate a delay in the timing of a tone in series of tones about 
Table 8

Weights of Two Models Correlated with $\Delta t$ for Conditions of Experiments 1 and 2

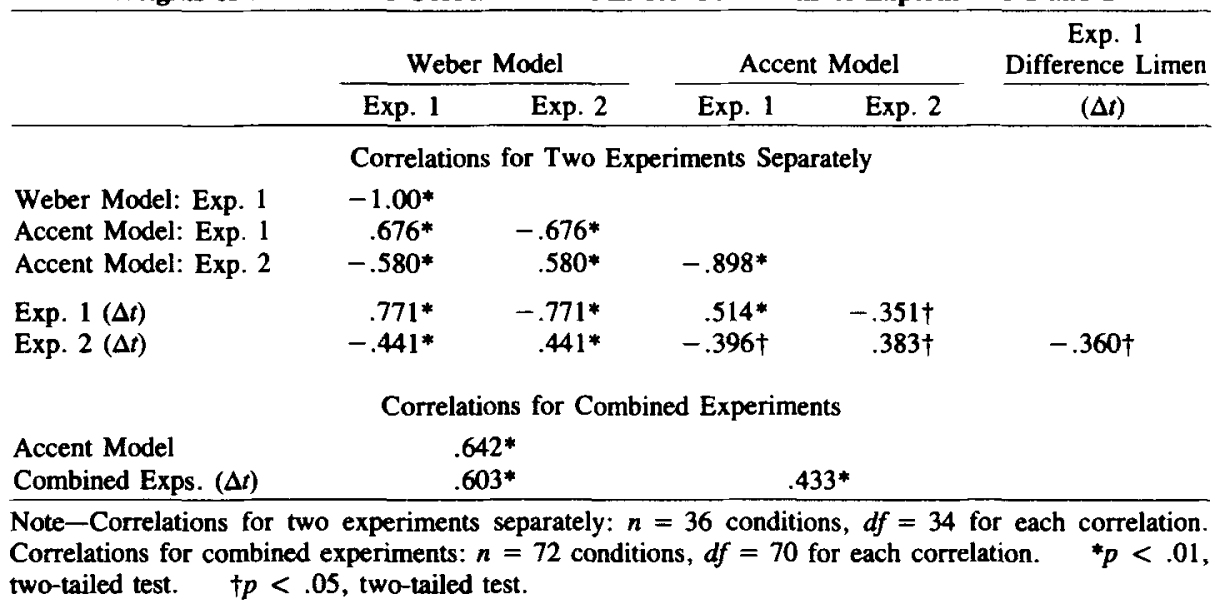

as well as they can when such tones define single temporal intervals, according to an older body of literature. When the sequences include combinations of long and short intervals, thereby generating rhythmic structures more complex than mere equal timing, there are some internal dependencies. For example, at slow tempos (short, long $=200,400 \mathrm{msec}$ ), a tone's temporal position is more easily discriminated when it separates 2 short intervals than when it separates any other combination of short and/or long intervals. At all tempos, discrimination for tones between 2 long intervals is difficult. At the fast tempo (short, long $=50,100 \mathrm{msec}$ ), there is an interesting asymmetry for the results on tones separating a short and a long interval. Delays of tones following long and preceding short intervals are better discriminated than those following short and preceding long intervals.

In addition to the model suggested above, we have attempted other models based on accent structure. None of them predicts the slow or musical tempo performance as well as does the average-interval Weber's law model. The major point is that temporal discrimination performance in complex, quasimusical contexts may well be modeled by an older psychophysical principle, at least for these noncycled sequences. It may be that appealing to cognitive representations of meter, accent, and the like will be unnecessary.

\section{REFERENCES}

ABEL, S. M. (1972). Duration discrimination of noise and tone bursts. Journal of the Acoustical Society of America, 51, 1219-1223.

Bharucha, J. J., \& Pryor, J. H. (1986). Disrupting the isochrony underlying thythm: An asymmetry in discrimination. Perception \& Psychophysics, 40, 137-141.

BolTz, M., \& Jones, M. R. (1986). Does rule recursion make melodies easier to produce? If not, what does? Cognitive Psychology, 18, $389-431$.

Bregman, A. S., CAmpbell, J. (1971). Primary auditory stream segregation and perception of order in rapid sequences of tones. Journal of Experimental Psychology, 89, 244-249.
Creelman, C. D. (1962). Human discrimination of auditory duration. Journal of the Acoustical Society of America, 34, 582-593.

DowLING, W. J. (1968). Rhythmic fission and perceptual organization of tone sequences. Unpublished doctoral dissertation, Harvard University, Cambridge, MA.

DowıJNG, W. J., \& HARWOOD, D. L. (1986). Music cognition. Orlando, FL: Academic Press.

ElLJoTt, L. (1962). Backward and forward masking of probe tones of different frequencies. Journal of the Acoustical Society of America, 34, 1116-1117.

Fitzgibbons, P. J., Pollatsek, A., * Thomas, I. B. (1974). Detection of temporal gaps within and between perceptual tonal groups. Perception \& Psychophysics, 16, 522-528.

Fourakis, M., \& Monahan, C. B. (1988). Effects of metrical foot structure on syllable timing. Language \& Speech, 31, 283-306.

Fraisse, P. (1963). The psychology of time. New York: Harper \& Row,

Fraisse, P. (1982). Rhythm and tempo. In D. Deutsch (Ed.), The psychology of music (pp. 149-180). New York: Academic Press.

GeTTY, D. J. (1975). Discrimination of short temporal intervals: A comparison of two models. Perception \& Psychophysics, 18, 1-8.

GRANT, K. W. (1987). Identification of intonation contours by normally hearing and profoundly hearing-impaired listeners. Journal of the Acoustical Society of America, 82, 1172-1178.

HANDEL, S., LAWSON, G. R. (1983). The contextual nature of thythmic interpretation. Perception \& Psychophysics, 34, 103-120.

HANDE L, S. , \& OshinsKy, J. S. (1981). The meter of syncopated auditory polyrhythms. Perception \& Psychophysics, 30, 1-9.

Hirsh, I. J., Monahan, C. B., Grant, K. W., Singh, P. G. (1990). Studies in auditory timing: 1 . Simple patterns. Perception \& Psychophysics, 47, 215-226.

KilleEN, P. R., Weiss, N. A. (1987). Optimal timing and the Weber function. Psychological Review, 94, 455-468.

Kristofferson, A. B. (1980). A quantal step function in duration discrimination. Perception \& Psychophysics, 27, 300-306.

LEVITT, H. (1971). Transformed up-down methods in psychophysics. Journal of the Acoustical Society of America, 49, 467-477.

Longuet-Higgins, H. C., LEE, C. S. (1982). The perception of musical rhythms. Perception, 11, 115-128.

MARTin, J. G. (1972). Rhythmic (hierarchical) versus serial structure in speech and other behavior. Psychological Review, 79, 487-509.

Monahan, C. B. (1984). Parallels between pitch and time: The determinants of musical space. Dissertation Abstracts Intemational, 45, 1942B. (University Microfilms No. 84-20, 214)

Monahan, C. B., \& Carterette, E. C. (1985). Pitch and duration as determinants of musical space. Music Perception, 3, 1-32.

Monahan, C. B., Kendall, R. A., \& Carterette, E. C. (1987). 
The effect of melodic and temporal contour on recognition memory for pitch change. Perception \& Psychophysics, 41, 576-600.

Pollack, I. (1967). Asynchrony: The perception of temporal gaps within periodic auditory pulse patterns. Journal of the Acoustical Society of America, 42, 1335-1340.

Povel, D.-J. (1981). Interval representation of simple temporal patterns. Journal of Experimental Psychology: Human Perception \& Performance, 7, 3-18.

Povel, D.-J. (1984). A theoretical framework for rhythm perception. Psychological Research, 45, 315-337.

Povel, D.-J., \& Essens, P. (1985). Perception of temporal patterns. Music Perception, 2, 411-440.

Povel, D.-J., OkKerman, H. (1981). Accents in equitone sequences. Perception \& Psychophysics, 30, 565-572.

ROYER, F. L., RoBIN, D. A. (1986). On the perceived unitization of repetitive auditory patterns. Perception \& Psychophysics, 39, 9-18.

Shields, J. L., McHugh, R., Martin, J. G. (1974). Reaction time to phoneme targets as a function of thythmic cues in continuous speech. Journal of Experimental Psychology, 102, 250-255.
Small, A. M., \& CAmpbell, R. A. (1962). Temporal differential sensitivity for auditory stimuli. American Joumal of Psychology, 75, 401-410.

STEEdman, M. J. (1977). The perception of musical rhythm and metre. Perception, 6, 555-570.

Sturges, P. T., \& MARTIN, J. G. (1974). Rhythmic structure in auditory temporal pattern perception and immediate memory. Journal of Experimental Psychology, 102, 377-383.

Thomassen, J. M. (1982). Melodic accent: Experiments and a tentative model. Joumal of the Acoustical Society of America, 71, 1596-1605.

VAN NOORDEN, L. P. A. S. (1975). Temporal coherence in the perception of tone sequences. Unpublished doctoral dissertation, Institute for Perceptual Research, Eindhoven, The Netherlands.

Yeston, M. (1976). The stratification of musical rhythm. New Haven, CT: Yale University Press.

(Manuscript received January 13, 1989; revision accepted for publication September 11, 1989.) 